\title{
A Comparison of Shape and Scale Estimators of the Two-Parameter Weibull Distribution
}

Florence George

Florida International University, fgeorge@fiu.edu

Follow this and additional works at: http://digitalcommons.wayne.edu/jmasm

Part of the Applied Statistics Commons, Social and Behavioral Sciences Commons, and the Statistical Theory Commons

\section{Recommended Citation}

George, Florence (2014) "A Comparison of Shape and Scale Estimators of the Two-Parameter Weibull Distribution," Journal of Modern Applied Statistical Methods: Vol. 13 : Iss. 1 , Article 3.

DOI: $10.22237 /$ jmasm/1398916920

Available at: http://digitalcommons.wayne.edu/jmasm/vol13/iss1/3 


\section{A Comparison of Shape and Scale Estimators of the Two-Parameter Weibull Distribution}

\section{Florence George}

Florida International University

Miami, FL

Weibull distributions are widely used in reliability and survival analysis. In this paper, different methods to estimate the shape and scale parameters of the two-parameter Weibull distribution have been reviewed and compared, based on the bias, mean square error and variance. Because a theoretical comparison is not possible, an extensive simulation study has been conducted to compare the performance of different estimators. Based on the simulation study it was observed that MLE consistently performs better than other methods.

Keywords: $\quad$ Two-parameter Weibull distribution, scale parameters, shape parameters

\section{Introduction}

The Weibull distribution is a commonly used model in reliability, life time and environmental data analysis. A considerable literature discussing the methods of estimation of Weibull parameters exists (Sharoon, et al., 2012; Saralees et al., 2011; Saralees et al., 2008) because of its applications in different fields. Kantar and Senoglu (2008) did a simulation comparison of different estimators for scale parameter when shape is known. Balakrishanan and Kateri (2008) showed the existence and uniqueness of maximum likelihood estimates (MLE) of Weibull distribution. Dubey (1967) derived the percentile estimators (Percentile 1) which uses 4 different percentiles to estimate the shape and scale parameters. Seki and Yokoyama (1993) proposed a simple and robust method that uses only two percentiles, $31^{\text {st }}$ and $63^{\text {rd }}$ percentile (Percentile 2) to estimate both parameters. Moment estimators (MOM) and median rank regression estimators (MRRS) are also commonly used in literature (Kantar and Senoglu, 2008) because of their

Dr. George is Assistant Professor in the Department of Mathematics and Statistics. Email herat:fgeorge@fiu.edu. 


\section{COMPARISON OF ESTIMATORS OF THE WEIBULL DISTRIBUTION}

easiness in computation. Existing methods (namely MLE, MOM, MRRS, Percentile 1, and Percentile 2) for estimating both shape and scale parameters of two-parameter Weibull distribution are here reviewed and compared. A simulation study has been conducted to compare the performance of these methods under same simulation conditions.

\section{Statistical Methodology}

The Weibull distribution has the probability density function, $f(x)=\alpha \beta^{-\alpha} x^{\alpha-1} e^{-\left(\frac{x}{\beta}\right)^{\alpha}}$ for $x \geq 0, \propto>0, \beta>0$, where $\propto$ is the shape parameter and $\beta$ is the scale parameter. The cumulative distribution function is given by

$$
F(x)=1-e^{-\left(\frac{x}{\beta}\right)^{\alpha}} \text { for } x \geq 0 \text {. }
$$

The distribution is reversed J-shaped when $\propto<<1$, exponential when $\propto c=1$ and bellshaped when $\propto>1$ (Kantar and Senoglu, 2008). Because of its wide-variety of shapes it is used extensively in practice for modeling real life data in different fields.

\section{Maximum Likelihood Estimators (MLE)}

The log-likelihood function of a random sample from the two-parameter Weibull distribution is given by

$\ln L=n \ln \alpha-n \alpha \ln \beta+(\alpha-1) \sum \ln x-\sum\left(\frac{x}{\beta}\right)^{\alpha}$

This will yield the following two score equations

$$
\begin{aligned}
& \frac{\partial \ln L}{\partial \beta}=-\frac{n \alpha}{\beta}+\frac{\alpha}{\beta^{\alpha+1}} \sum x^{a}=0 \text { and } \\
& \frac{\partial \ln L}{\partial \alpha}=-\frac{n}{\alpha}-n \ln \beta+\sum \ln x+\frac{\ln \beta}{\beta^{a}} \sum x^{\alpha}-\frac{1}{\beta^{a}} \sum x^{\alpha} \ln x=0
\end{aligned}
$$

The above two equations can be solved numerically to obtain MLEs. 


\section{FLORENCE GEORGE}

\section{Moment Estimators (MOM)}

The moment estimators are obtained by equating the population moments to the corresponding sample moments. The first and second moments of Weibull distribution are respectively

$\mu_{1}^{\prime}=\beta \Gamma\left(1+\alpha^{-1}\right)$ and

$\mu_{2}^{\prime}=\beta^{2} \Gamma\left(1+2 \alpha^{-1}\right)$

The first two moments from the sample are $m_{1}^{\prime}=\frac{1}{n} \sum x$ and $m_{2}^{\prime}=\frac{1}{n} \sum x^{2}$.

The moment estimates are obtained by solving the following two equations

$m_{1}^{\prime}=\beta \Gamma\left(1+\alpha^{-1}\right)$

$m_{2}^{\prime}=\beta^{2} \Gamma\left(1+2 \alpha^{-1}\right)$

\section{Median Rank Regression Estimators (MRRS)}

MRR is a procedure for estimating the Weibull parameters by fitting a least squares regression line through the points on a probability plot. Thus,

$\log (1-F(x))=-\left(\frac{x}{\beta}\right)^{\alpha}$ and hence

$\log (\log (1-F(x)))=\alpha \log x=\alpha \log \beta$.

This is now a linear model and method of least squares can be used to estimate $\alpha$ and $\beta$. The sample data are first sorted in ascending order and then following Abernethy (2006), the distribution function, $F\left(x_{i}\right)$ is approximated for each point $\left(x_{i}\right)$ in the sorted sample as $F\left(x_{i}\right)=\frac{i-0.3}{n+0.4}$, where $I$ is the ascending rank of the data point $x_{i}$ 


\section{COMPARISON OF ESTIMATORS OF THE WEIBULL DISTRIBUTION}

\section{Percentile Estimators (Percentile 1)}

Percentile estimators for both shape and scale parameters were derived by Dubey (1967). He proposed an estimator based on $17^{\text {th }}$ and $97^{\text {th }}$ percentiles for shape parameter and one based on $40^{\text {th }}$ and $82^{\text {nd }}$ percentile for scale parameter. The formulae for the shape and scale percentile estimators are presented here; for details refer to Dubey (1967). Let $p_{1}=0.1673$ and $p_{2}=0.9737$. Define $k_{1}=$ $\log \left(-\log \left(1-p_{1}\right)\right)-\log \left(-\log \left(1-p_{2}\right)\right)$. Let $y_{1}$ and $y_{2}$ represent the $100 p_{1}$ th percentile from the data. Then

$$
\hat{\alpha}=\frac{-k_{1}}{\log \left(y_{1}\right)-\log \left(y_{2}\right)}
$$

Similarly to estimate $\beta$, define $p_{3}=0.3978$ and $p_{4}=0.8211$. Let $k_{2}=$ $\log \left(-\log \left(1-p_{3}\right)\right)-\log \left(-\log \left(1-p_{4}\right)\right) ; k_{3}=-\log \left(1-p_{3}\right)$ and $w=1-\frac{\log \left(k_{3}\right)}{k_{2}}$. Let $y_{3}$ and $y_{4}$ represent the $100 p_{3}$ th and $100 p_{4}$ th percentile from the data. Then

$$
\beta=\exp \left(w \log \left(y_{3}\right)+(1-w) \log \left(y_{4}\right) .\right.
$$

\section{Improved Percentile Estimators (Percentile 2)}

Seki and Yokoyama (1993) proposed this simple and robust method that uses only two percentiles, $31 \mathrm{st}$ and $63^{\text {rd }}$ percentile to estimate $\alpha$ and $\beta$. The Weibull cumulative distribution function is given by

$$
F(x)=1-e^{-\left(\frac{x}{\beta}\right)^{\alpha}} \text { for } x \geq 0 .
$$

Hence the $100 p$ th percentile of the Weibull distribution can be written as $x_{p}=\beta(-\log (1-p))^{1 / \beta}$. Then the $100\left(1-e^{-1}\right)=63.2^{\text {th }}$ percentile is $x_{0.632}=\beta$ for any Weibull distribution. This can be used to compute $\hat{\beta}$. Therefore, the estimate of the shape parameter can be obtained as $\hat{\alpha}=\frac{\log (-\log (1-p))}{\log \left(\frac{x_{p}}{x_{0.632}}\right)}$. Seki and

Yokoyama (1993) approximated the numerator of this estimator as -1 and then obtained $p=0.31$, approximately, to obtain $\hat{\alpha}$. 


\section{FLORENCE GEORGE}

\section{Simulation Study}

A simulation study has been conducted to explore the performances of the different methods discussed in this article.

\section{Simulation Technique}

The main objective of this study is to compare the performance of five different methods to estimate the shape and scale parameters of two-parameter Weibull distribution. Weibull distribution with parameters scale $=10$ and shape $=0.5,1$, $1.5,2,3$ and 4 were used to generate 5,000 samples of sizes $n=5,10,20,30,50$ and 100. The estimates are compared using the values of average bias, mean squared error (MSE) and variance. The simulation was done using statistical software R version 2.15.2.

\section{Results and Discussion}

The results of the simulation are shown in Tables 1 to 3. The bias and MSEs from Weibull $(10,0.5)$ and Weibull $(10,3)$ are also presented in Figures 1 to 4. From Tables 1 to 3, it can be observed that as sample size increases, bias, MSE and variance decrease. For small sample size, the performance of methods differs significantly. For all methods, absolute bias, MSE and variance decrease as sample size increases. It can be observed from Tables 1 to 3 and Figures 1 to 4 , in almost all cases MLE performed better than the other 4 methods and percentile method-1 performed the worst. In some situations, MRRS also performs well, especially for shape estimates. It can also be observed that both percentile estimators perform poorly in estimation of shape. There is no consistency in the performance of estimates by the method of moments. Because MLE is performing consistently better than the other 4 methods practitioners are encouraged to use MLE whenever possible. 


\section{COMPARISON OF ESTIMATORS OF THE WEIBULL DISTRIBUTION}

Table 1. Bias, Variance and MSE of both Scale and Shape estimates $\alpha=10$ and $\beta=0.5 ; 1$

\begin{tabular}{|c|c|c|c|c|c|c|c|c|c|c|c|c|}
\hline \multirow[b]{2}{*}{$\alpha, \beta$} & \multirow[b]{2}{*}{$n$} & & \multicolumn{5}{|c|}{ Scale } & \multicolumn{5}{|c|}{ Shape } \\
\hline & & & MLE & MOM & MRRS & Perctle1 & Perctle2 & MLE & MOM & MRRS & Perctle1 & Perctle2 \\
\hline \multirow{18}{*}{$10,0.5$} & \multirow{3}{*}{5} & Bias & 2.929 & 6.216 & 5.361 & 5.340 & 4.394 & 0.219 & 0.351 & 0.025 & 0.661 & 0.497 \\
\hline & & Vars & 156.930 & 224.302 & 219.866 & 282.068 & 197.209 & 0.147 & 0.092 & 0.085 & 5.231 & 0.313 \\
\hline & & MSEs & 165.511 & 262.939 & 248.610 & 310.581 & 216.516 & 0.195 & 0.216 & 0.085 & 5.668 & 0.560 \\
\hline & \multirow{3}{*}{10} & Bias & 1.525 & 4.606 & 2.998 & 2.334 & 1.773 & 0.085 & 0.218 & -0.015 & 0.283 & 0.193 \\
\hline & & Vars & 65.147 & 96.815 & 84.821 & 110.433 & 73.834 & 0.031 & 0.033 & 0.026 & 0.349 & 0.050 \\
\hline & & MSEs & 67.473 & 118.026 & 93.811 & 115.879 & 76.977 & 0.038 & 0.081 & 0.026 & 0.429 & 0.087 \\
\hline & \multirow{3}{*}{20} & Bias & 0.705 & 3.217 & 1.643 & 0.979 & 0.656 & 0.036 & 0.136 & -0.021 & 0.112 & 0.079 \\
\hline & & Vars & 25.634 & 40.337 & 32.389 & 43.623 & 30.013 & 0.010 & 0.016 & 0.012 & 0.069 & 0.015 \\
\hline & & MSEs & 26.131 & 50.689 & 35.088 & 44.582 & 30.444 & 0.012 & 0.035 & 0.013 & 0.082 & 0.021 \\
\hline & \multirow{3}{*}{30} & Bias & 0.556 & 2.621 & 1.258 & 0.707 & 0.442 & 0.026 & 0.107 & -0.017 & 0.072 & 0.054 \\
\hline & & Vars & 17.111 & 25.216 & 20.965 & 26.613 & 19.722 & 0.006 & 0.013 & 0.008 & 0.036 & 0.009 \\
\hline & & MSEs & 17.420 & 32.084 & 22.548 & 27.113 & 19.917 & 0.007 & 0.024 & 0.008 & 0.041 & 0.012 \\
\hline & \multirow{3}{*}{50} & Bias & 0.283 & 1.912 & 0.783 & 0.405 & 0.207 & 0.014 & 0.076 & -0.016 & 0.039 & 0.034 \\
\hline & & Vars & 9.585 & 15.077 & 11.172 & 15.220 & 11.347 & 0.003 & 0.008 & 0.005 & 0.017 & 0.005 \\
\hline & & MSEs & 9.665 & 18.731 & 11.785 & 15.384 & 11.390 & 0.004 & 0.014 & 0.005 & 0.019 & 0.006 \\
\hline & \multirow{3}{*}{100} & Bias & 0.174 & 1.257 & 0.487 & 0.236 & 0.158 & 0.008 & 0.048 & -0.011 & 0.021 & 0.018 \\
\hline & & Vars & 4.664 & 8.153 & 5.320 & 7.373 & 5.727 & 0.002 & 0.005 & 0.003 & 0.007 & 0.002 \\
\hline & & MSEs & 4.694 & 9.734 & 5.557 & 7.429 & 5.752 & 0.002 & 0.008 & 0.003 & 0.008 & 0.003 \\
\hline \multirow{18}{*}{10,1} & \multirow{3}{*}{5} & Bias & 0.284 & 0.312 & 1.232 & 0.485 & 0.304 & 0.443 & 0.353 & 0.049 & 1.297 & 0.926 \\
\hline & & Vars & 23.380 & 23.097 & 27.835 & 29.087 & 24.408 & 0.621 & 0.411 & 0.347 & 10.932 & 1.147 \\
\hline & & MSEs & 23.461 & 23.194 & 29.353 & 29.322 & 24.501 & 0.817 & 0.535 & 0.349 & 12.614 & 2.005 \\
\hline & \multirow{3}{*}{10} & Bias & 0.190 & 0.222 & 0.840 & 0.223 & 0.060 & 0.173 & 0.173 & -0.032 & 0.507 & 0.371 \\
\hline & & Vars & 11.082 & 11.192 & 12.944 & 15.822 & 12.013 & 0.127 & 0.108 & 0.106 & 0.991 & 0.199 \\
\hline & & MSEs & 11.118 & 11.241 & 13.650 & 15.872 & 12.016 & 0.157 & 0.137 & 0.107 & 1.247 & 0.336 \\
\hline & \multirow{3}{*}{20} & Bias & 0.072 & 0.100 & 0.486 & 0.067 & -0.047 & 0.074 & 0.087 & -0.042 & 0.225 & 0.158 \\
\hline & & Vars & 5.576 & 5.707 & 6.335 & 8.386 & 6.348 & 0.043 & 0.046 & 0.048 & 0.281 & 0.063 \\
\hline & & MSEs & 5.581 & 5.717 & 6.571 & 8.390 & 6.350 & 0.049 & 0.053 & 0.050 & 0.331 & 0.088 \\
\hline & \multirow{3}{*}{30} & Bias & 0.075 & 0.098 & 0.412 & 0.049 & -0.006 & 0.049 & 0.062 & -0.040 & 0.141 & 0.108 \\
\hline & & Vars & 3.671 & 3.796 & 4.182 & 5.412 & 4.340 & 0.025 & 0.029 & 0.032 & 0.134 & 0.037 \\
\hline & & MSEs & 3.676 & 3.805 & 4.351 & 5.414 & 4.340 & 0.027 & 0.033 & 0.033 & 0.154 & 0.048 \\
\hline & \multirow{3}{*}{50} & Bias & 0.050 & 0.062 & 0.281 & 0.028 & -0.010 & 0.029 & 0.039 & -0.031 & 0.087 & 0.069 \\
\hline & & Vars & 2.173 & 2.269 & 2.426 & 3.332 & 2.635 & 0.014 & 0.018 & 0.020 & 0.072 & 0.020 \\
\hline & & MSEs & 2.175 & 2.273 & 2.504 & 3.332 & 2.635 & 0.015 & 0.019 & 0.021 & 0.080 & 0.025 \\
\hline & \multirow{3}{*}{100} & Bias & 0.032 & 0.034 & 0.179 & 0.012 & 0.002 & 0.016 & 0.021 & -0.021 & 0.045 & 0.037 \\
\hline & & Vars & 1.117 & 1.178 & 1.232 & 1.698 & 1.349 & 0.007 & 0.010 & 0.010 & 0.031 & 0.010 \\
\hline & & MSEs & 1.118 & 1.179 & 1.264 & 1.698 & 1.349 & 0.007 & 0.010 & 0.011 & 0.033 & 0.011 \\
\hline
\end{tabular}




\section{FLORENCE GEORGE}

Table 2. Bias, Variance and MSE of both Scale and Shape estimates $\alpha=10$ and $\beta=1.5 ; 2$

\begin{tabular}{|c|c|c|c|c|c|c|c|c|c|c|c|c|}
\hline \multirow[b]{2}{*}{$\alpha, \beta$} & \multirow[b]{2}{*}{$n$} & & \multicolumn{5}{|c|}{ Scale } & \multicolumn{5}{|c|}{ Shape } \\
\hline & & & MLE & MOM & MRRS & Perctle1 & Perctle2 & MLE & MOM & MRRS & Perctle1 & Perctle2 \\
\hline \multirow{18}{*}{$10,1.5$} & \multirow{3}{*}{5} & Bias & -0.081 & -0.149 & 0.513 & -0.149 & -0.226 & 0.650 & 0.398 & 0.059 & 2.045 & 1.298 \\
\hline & & Vars & 9.333 & 9.331 & 10.477 & 11.069 & 9.546 & 1.374 & 0.947 & 0.713 & 47.203 & 2.365 \\
\hline & & MSEs & 9.340 & 9.353 & 10.740 & 11.091 & 9.597 & 1.797 & 1.106 & 0.717 & 51.386 & 4.050 \\
\hline & \multirow{3}{*}{10} & Bias & 0.038 & -0.002 & 0.451 & -0.016 & -0.092 & 0.257 & 0.171 & -0.048 & 0.755 & 0.537 \\
\hline & & Vars & 4.948 & 4.986 & 5.542 & 6.870 & 5.493 & 0.275 & 0.231 & 0.232 & 2.331 & 0.427 \\
\hline & & MSEs & 4.950 & 4.986 & 5.746 & 6.870 & 5.501 & 0.342 & 0.260 & 0.235 & 2.901 & 0.715 \\
\hline & \multirow{3}{*}{20} & Bias & 0.039 & 0.016 & 0.317 & 0.016 & -0.046 & 0.114 & 0.081 & -0.065 & 0.339 & 0.248 \\
\hline & & Vars & 2.508 & 2.517 & 2.763 & 3.802 & 2.851 & 0.097 & 0.090 & 0.109 & 0.606 & 0.144 \\
\hline & & MSEs & 2.509 & 2.517 & 2.863 & 3.802 & 2.853 & 0.110 & 0.096 & 0.113 & 0.721 & 0.205 \\
\hline & \multirow{3}{*}{30} & Bias & 0.014 & -0.003 & 0.227 & -0.006 & -0.064 & 0.075 & 0.054 & -0.054 & 0.209 & 0.167 \\
\hline & & Vars & 1.669 & 1.677 & 1.816 & 2.439 & 1.947 & 0.055 & 0.054 & 0.074 & 0.324 & 0.083 \\
\hline & & MSEs & 1.669 & 1.677 & 1.867 & 2.439 & 1.952 & 0.061 & 0.057 & 0.077 & 0.368 & 0.111 \\
\hline & \multirow{3}{*}{50} & Bias & -0.008 & -0.019 & 0.145 & -0.021 & -0.050 & 0.041 & 0.030 & -0.050 & 0.121 & 0.099 \\
\hline & & Vars & 0.973 & 0.979 & 1.048 & 1.486 & 1.166 & 0.031 & 0.032 & 0.045 & 0.155 & 0.048 \\
\hline & & MSEs & 0.973 & 0.979 & 1.069 & 1.486 & 1.168 & 0.033 & 0.033 & 0.048 & 0.170 & 0.058 \\
\hline & \multirow{3}{*}{100} & Bias & 0.003 & -0.004 & 0.102 & -0.020 & -0.027 & 0.022 & 0.016 & -0.032 & 0.067 & 0.054 \\
\hline & & Vars & 0.493 & 0.495 & 0.533 & 0.748 & 0.591 & 0.014 & 0.016 & 0.023 & 0.070 & 0.021 \\
\hline & & MSEs & 0.493 & 0.495 & 0.543 & 0.749 & 0.592 & 0.015 & 0.016 & 0.024 & 0.074 & 0.024 \\
\hline \multirow{18}{*}{10,2} & \multirow{3}{*}{5} & Bias & -0.102 & -0.123 & 0.339 & -0.200 & -0.263 & 0.868 & 0.510 & 0.089 & 2.499 & 1.673 \\
\hline & & Vars & 5.328 & 5.376 & 5.763 & 6.306 & 5.441 & 2.301 & 1.833 & 1.321 & 43.582 & 3.716 \\
\hline & & MSEs & 5.339 & 5.391 & 5.878 & 6.345 & 5.511 & 3.055 & 2.093 & 1.329 & 49.828 & 6.516 \\
\hline & \multirow{3}{*}{10} & Bias & -0.049 & -0.065 & 0.254 & -0.132 & -0.189 & 0.338 & 0.191 & -0.060 & 1.024 & 0.699 \\
\hline & & Vars & 2.797 & 2.830 & 3.090 & 3.811 & 3.062 & 0.459 & 0.390 & 0.394 & 4.718 & 0.699 \\
\hline & & MSEs & 2.799 & 2.834 & 3.154 & 3.829 & 3.098 & 0.573 & 0.427 & 0.398 & 5.767 & 1.187 \\
\hline & \multirow{3}{*}{20} & Bias & -0.041 & -0.051 & 0.157 & -0.089 & -0.136 & 0.151 & 0.087 & -0.080 & 0.473 & 0.331 \\
\hline & & Vars & 1.359 & 1.368 & 1.461 & 2.068 & 1.560 & 0.171 & 0.159 & 0.197 & 1.296 & 0.262 \\
\hline & & MSEs & 1.361 & 1.371 & 1.486 & 2.076 & 1.578 & 0.194 & 0.166 & 0.203 & 1.520 & 0.371 \\
\hline & \multirow{3}{*}{30} & Bias & -0.004 & -0.009 & 0.157 & -0.023 & -0.059 & 0.096 & 0.054 & -0.081 & 0.275 & 0.222 \\
\hline & & Vars & 0.911 & 0.915 & 0.991 & 1.347 & 1.059 & 0.096 & 0.090 & 0.125 & 0.540 & 0.142 \\
\hline & & MSEs & 0.911 & 0.915 & 1.016 & 1.348 & 1.062 & 0.105 & 0.093 & 0.131 & 0.615 & 0.191 \\
\hline & \multirow{3}{*}{50} & Bias & -0.007 & -0.010 & 0.110 & -0.028 & -0.049 & 0.059 & 0.035 & -0.063 & 0.166 & 0.141 \\
\hline & & Vars & 0.543 & 0.546 & 0.596 & 0.829 & 0.654 & 0.055 & 0.054 & 0.080 & 0.269 & 0.084 \\
\hline & & MSEs & 0.543 & 0.546 & 0.608 & 0.830 & 0.656 & 0.059 & 0.055 & 0.084 & 0.297 & 0.104 \\
\hline & \multirow{3}{*}{100} & Bias & 0.001 & -0.002 & 0.071 & -0.020 & -0.022 & 0.031 & 0.019 & -0.040 & 0.088 & 0.073 \\
\hline & & Vars & 0.276 & 0.276 & 0.296 & 0.427 & 0.332 & 0.025 & 0.025 & 0.041 & 0.120 & 0.037 \\
\hline & & MSEs & 0.276 & 0.276 & 0.301 & 0.427 & 0.333 & 0.026 & 0.026 & 0.042 & 0.128 & 0.043 \\
\hline
\end{tabular}




\section{COMPARISON OF ESTIMATORS OF THE WEIBULL DISTRIBUTION}

Table 3. Bias, Variance and MSE of both Scale and Shape estimates $\alpha=10$ and $\beta=3 ; 4$

\begin{tabular}{|c|c|c|c|c|c|c|c|c|c|c|c|c|}
\hline \multirow[b]{2}{*}{$\alpha, \beta$} & \multirow[b]{2}{*}{$n$} & & \multicolumn{5}{|c|}{ Scale } & \multicolumn{5}{|c|}{ Shape } \\
\hline & & & MLE & MOM & MRRS & Perctle1 & Perctle2 & MLE & MOM & MRRS & Perctle1 & Perctle2 \\
\hline \multirow{18}{*}{10,3} & \multirow{3}{*}{5} & Bias & -0.166 & -0.138 & 0.122 & -0.275 & -0.320 & 1.295 & 0.767 & 0.131 & 3.899 & 2.417 \\
\hline & & Vars & 2.392 & 2.439 & 2.515 & 2.865 & 2.489 & 5.768 & 5.041 & 3.285 & 243.739 & 8.976 \\
\hline & & MSEs & 2.419 & 2.458 & 2.530 & 2.941 & 2.591 & 7.446 & 5.629 & 3.302 & 258.938 & 14.818 \\
\hline & \multirow{3}{*}{10} & Bias & -0.053 & -0.037 & 0.147 & -0.129 & -0.152 & 0.505 & 0.276 & -0.102 & 1.461 & 1.048 \\
\hline & & Vars & 1.221 & 1.234 & 1.290 & 1.659 & 1.352 & 1.100 & 0.998 & 0.911 & 9.896 & 1.722 \\
\hline & & MSEs & 1.224 & 1.235 & 1.312 & 1.676 & 1.374 & 1.354 & 1.074 & 0.922 & 12.032 & 2.820 \\
\hline & \multirow{3}{*}{20} & Bias & -0.017 & -0.009 & 0.120 & -0.060 & -0.070 & 0.218 & 0.113 & -0.134 & 0.689 & 0.487 \\
\hline & & Vars & 0.602 & 0.605 & 0.649 & 0.896 & 0.690 & 0.374 & 0.359 & 0.428 & 2.594 & 0.572 \\
\hline & & MSEs & 0.602 & 0.605 & 0.663 & 0.900 & 0.695 & 0.422 & 0.372 & 0.445 & 3.070 & 0.810 \\
\hline & \multirow{3}{*}{30} & Bias & -0.022 & -0.017 & 0.082 & -0.049 & -0.057 & 0.153 & 0.084 & -0.110 & 0.452 & 0.341 \\
\hline & & Vars & 0.415 & 0.417 & 0.442 & 0.618 & 0.487 & 0.230 & 0.227 & 0.294 & 1.387 & 0.343 \\
\hline & & MSEs & 0.416 & 0.417 & 0.448 & 0.620 & 0.491 & 0.253 & 0.234 & 0.306 & 1.592 & 0.459 \\
\hline & \multirow{3}{*}{50} & Bias & -0.020 & -0.017 & 0.058 & -0.039 & -0.044 & 0.083 & 0.044 & -0.101 & 0.254 & 0.209 \\
\hline & & Vars & 0.243 & 0.243 & 0.259 & 0.358 & 0.288 & 0.121 & 0.123 & 0.185 & 0.643 & 0.183 \\
\hline & & MSEs & 0.243 & 0.243 & 0.262 & 0.360 & 0.290 & 0.128 & 0.125 & 0.196 & 0.707 & 0.226 \\
\hline & \multirow{3}{*}{100} & Bias & 0.000 & 0.001 & 0.047 & -0.003 & -0.014 & 0.035 & 0.014 & -0.075 & 0.098 & 0.096 \\
\hline & & Vars & 0.118 & 0.118 & 0.128 & 0.186 & 0.144 & 0.058 & 0.059 & 0.092 & 0.265 & 0.087 \\
\hline & & MSEs & 0.118 & 0.118 & 0.130 & 0.186 & 0.144 & 0.059 & 0.059 & 0.098 & 0.274 & 0.096 \\
\hline \multirow{18}{*}{10,4} & \multirow{3}{*}{5} & Bias & -0.125 & -0.081 & 0.091 & -0.230 & -0.258 & 1.716 & 1.051 & 0.164 & 4.996 & 3.156 \\
\hline & & Vars & 1.388 & 1.411 & 1.442 & 1.683 & 1.471 & 9.001 & 7.677 & 5.003 & 141.435 & 13.999 \\
\hline & & MSEs & 1.404 & 1.418 & 1.451 & 1.736 & 1.537 & 11.945 & 8.782 & 5.029 & 166.393 & 23.962 \\
\hline & \multirow{3}{*}{10} & Bias & -0.078 & -0.054 & 0.076 & -0.140 & -0.156 & 0.686 & 0.401 & -0.131 & 2.017 & 1.402 \\
\hline & & Vars & 0.670 & 0.675 & 0.709 & 0.913 & 0.747 & 2.063 & 1.999 & 1.723 & 16.360 & 3.181 \\
\hline & & MSEs & 0.676 & 0.678 & 0.714 & 0.932 & 0.772 & 2.535 & 2.159 & 1.740 & 20.429 & 5.146 \\
\hline & \multirow{3}{*}{20} & Bias & -0.033 & -0.021 & 0.070 & -0.056 & -0.076 & 0.305 & 0.166 & -0.174 & 0.875 & 0.659 \\
\hline & & Vars & 0.362 & 0.364 & 0.391 & 0.545 & 0.420 & 0.691 & 0.695 & 0.774 & 4.648 & 1.023 \\
\hline & & MSEs & 0.363 & 0.365 & 0.396 & 0.548 & 0.426 & 0.783 & 0.723 & 0.804 & 5.414 & 1.457 \\
\hline & \multirow{3}{*}{30} & Bias & -0.024 & -0.016 & 0.055 & -0.038 & -0.055 & 0.194 & 0.107 & -0.156 & 0.549 & 0.444 \\
\hline & & Vars & 0.229 & 0.230 & 0.245 & 0.339 & 0.271 & 0.388 & 0.411 & 0.523 & 2.300 & 0.567 \\
\hline & & MSEs & 0.229 & 0.230 & 0.248 & 0.341 & 0.274 & 0.425 & 0.423 & 0.547 & 2.601 & 0.764 \\
\hline & \multirow{3}{*}{50} & Bias & -0.016 & -0.011 & 0.042 & -0.025 & -0.035 & 0.112 & 0.060 & -0.132 & 0.322 & 0.271 \\
\hline & & Vars & 0.138 & 0.139 & 0.149 & 0.209 & 0.163 & 0.224 & 0.237 & 0.323 & 1.087 & 0.342 \\
\hline & & MSEs & 0.138 & 0.139 & 0.151 & 0.209 & 0.165 & 0.237 & 0.241 & 0.341 & 1.190 & 0.415 \\
\hline & \multirow{3}{*}{100} & Bias & -0.013 & -0.011 & 0.023 & -0.021 & -0.025 & 0.060 & 0.036 & -0.087 & 0.156 & 0.147 \\
\hline & & Vars & 0.066 & 0.067 & 0.071 & 0.104 & 0.082 & 0.108 & 0.116 & 0.174 & 0.478 & 0.157 \\
\hline & & MSEs & 0.066 & 0.067 & 0.072 & 0.105 & 0.083 & 0.111 & 0.118 & 0.181 & 0.503 & 0.178 \\
\hline
\end{tabular}




\section{FLORENCE GEORGE}
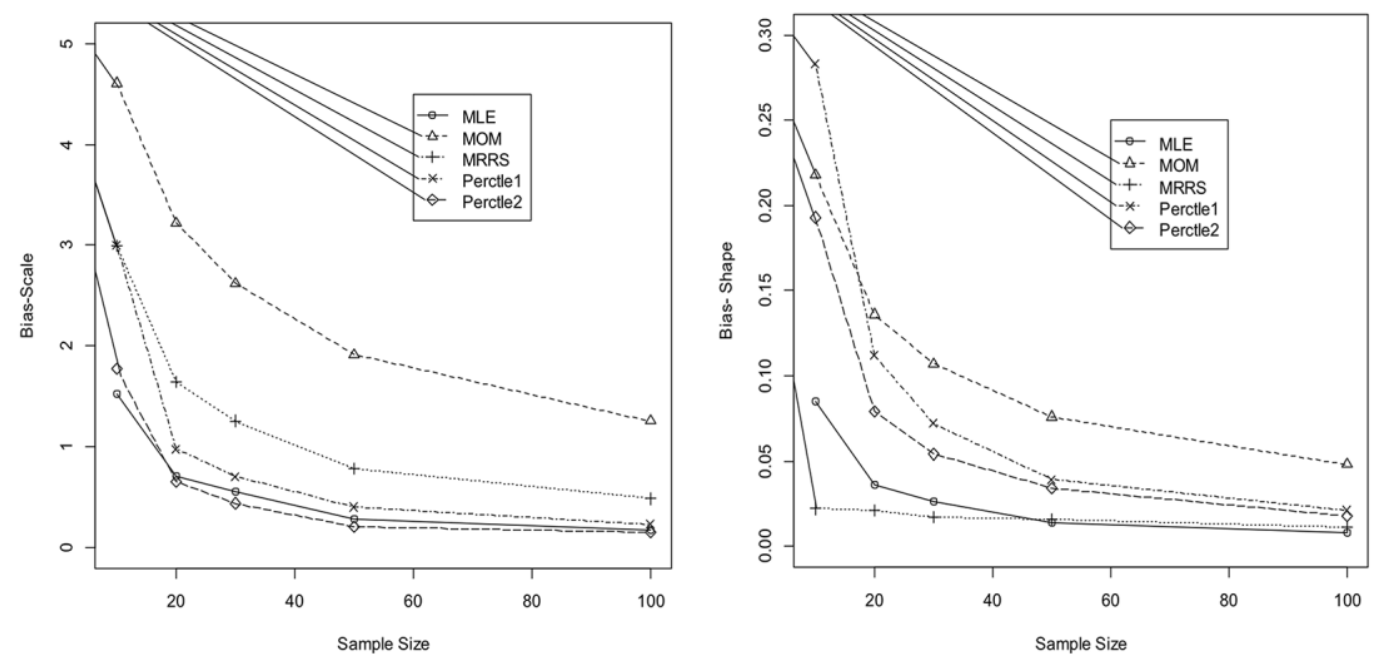

Figure 1. Absolute Bias of Scale parameter estimate (left), Shape parameter estimate (right) vs. Sample size from Weibull $(10,0.5)$
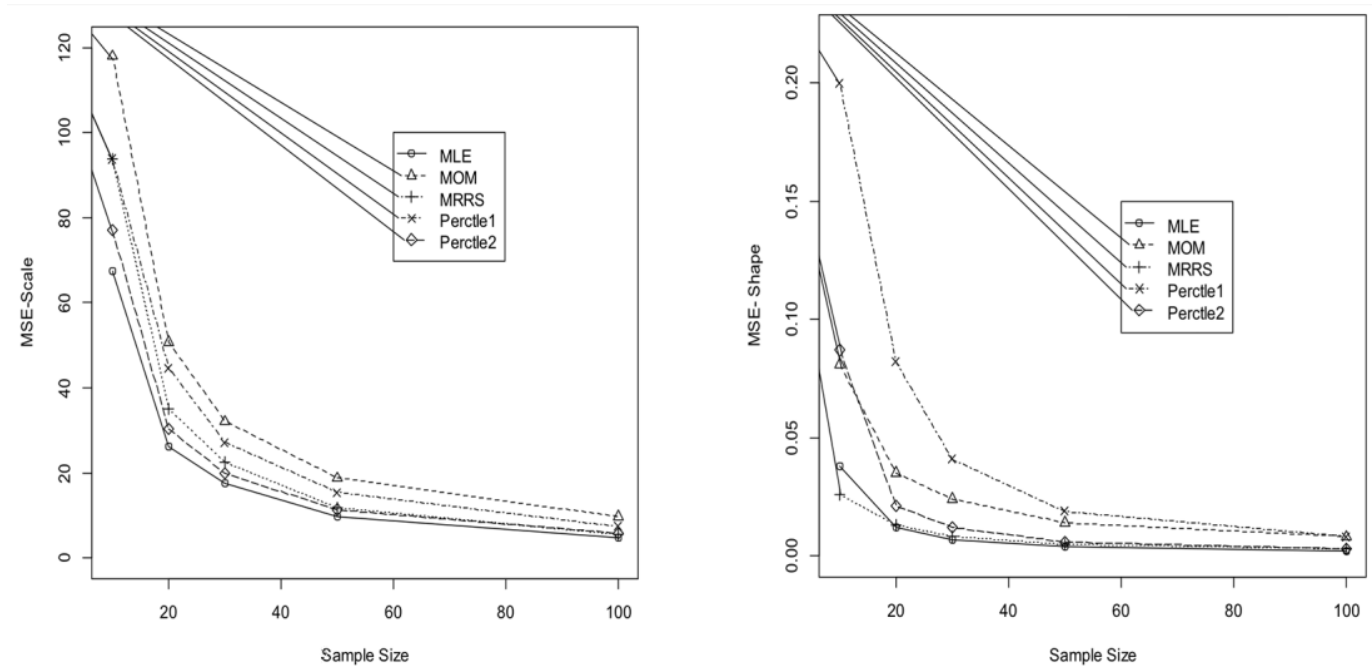

Figure 2. MSE of Scale parameter estimate (left), Shape parameter estimate (right) vs. Sample size from Weibull $(10,0.5)$ 


\section{COMPARISON OF ESTIMATORS OF THE WEIBULL DISTRIBUTION}
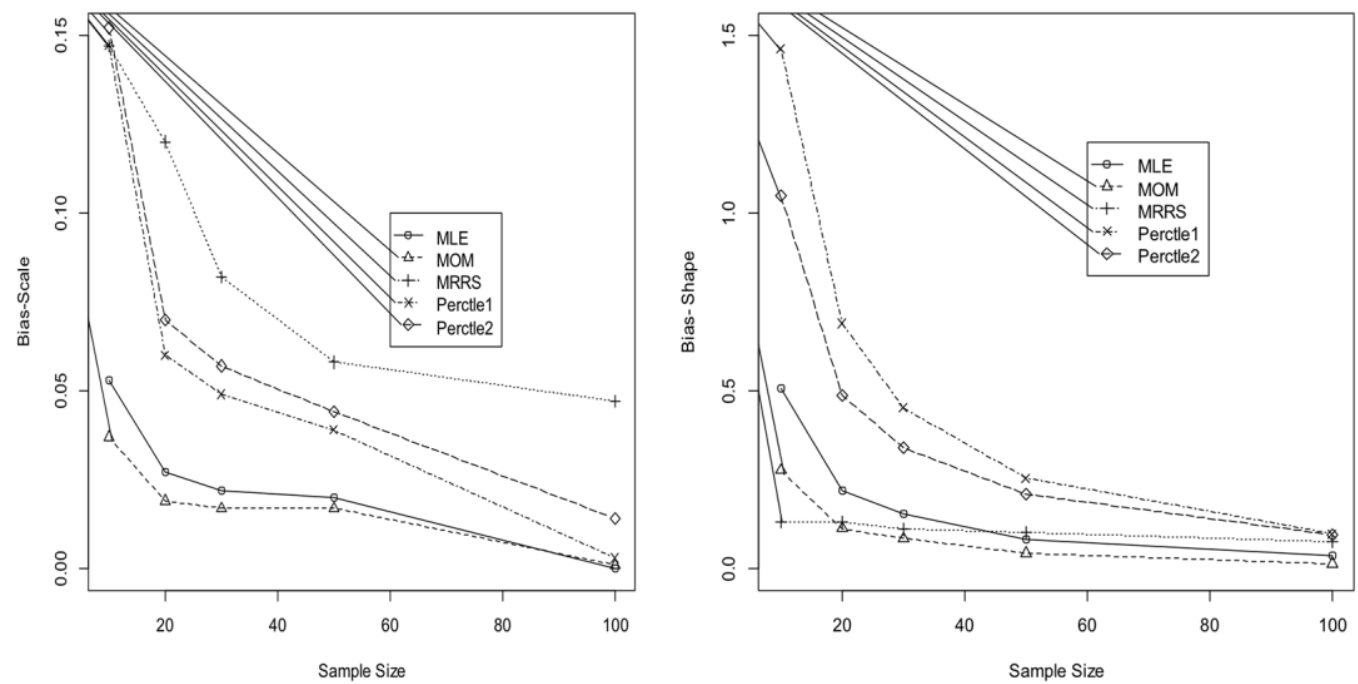

Figure 3. Absolute Bias of Scale parameter estimate (left), Shape parameter estimate (right) vs. Sample size from Weibull $(10,3)$
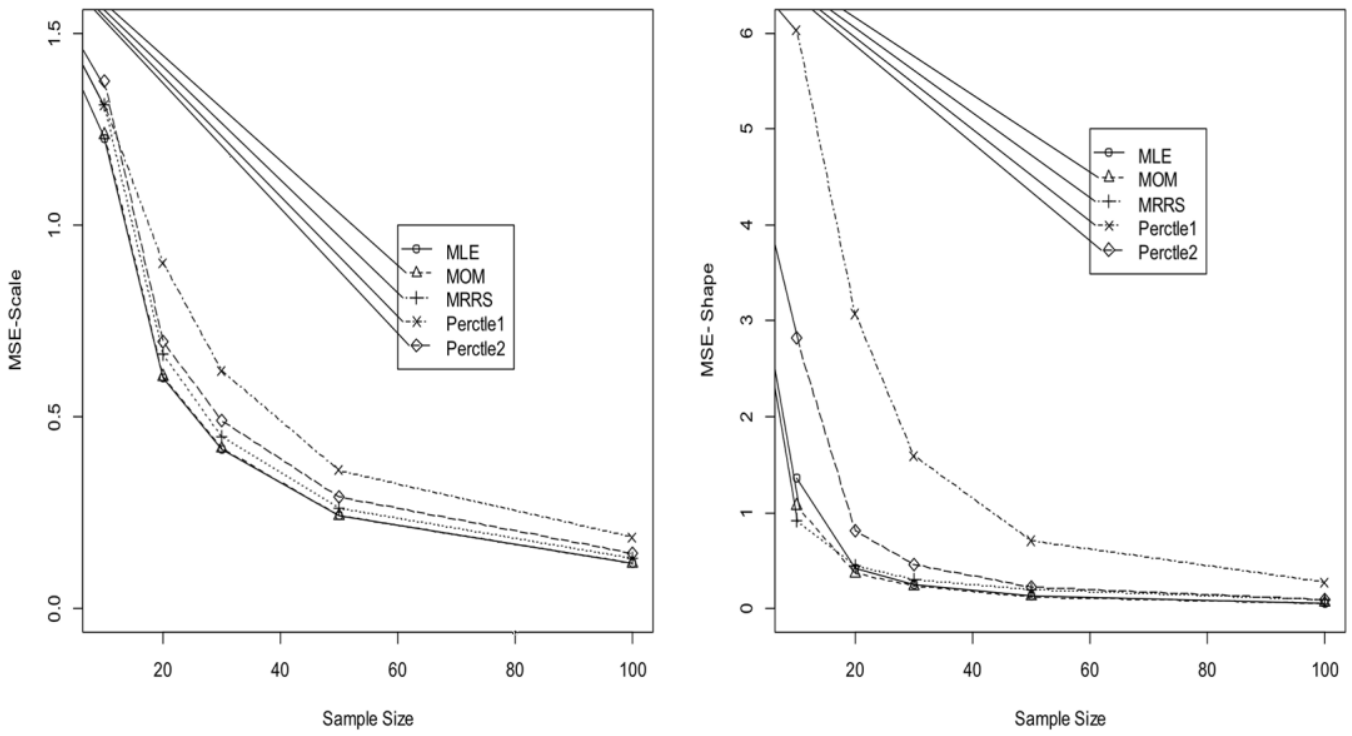

Figure 4. MSE of Scale parameter estimate (left), Shape parameter estimate (right) vs. Sample size from Weibull $(10,3)$ 


\section{FLORENCE GEORGE}

\section{Example}

Many researchers modeled wind data using the Weibull distribution (Dorvlo, 2002; Weisser, 2003; Celik, 2003). The five methods with an example in Battacharya and Bhattacharjee (2010) will be discussed next. This example provides the average monthly wind speed $(\mathrm{m} / \mathrm{s})$ of Kolkata from $1^{\text {st }}$ March 2009 to $31^{\text {st }}$ March 2009. Table 4 presents the data set.

The estimates of the two-parameter Weibull distribution obtained by fitting to the data using the methods discussed in the article are given in the Table 5. It look like the Percentile 1 and Percentile 2 estimates are at the extreme ends and the MLE estimates lie somewhat between the values of other estimates.

Table 4. Average daily wind speed in Kolkata during March 2009.

\begin{tabular}{lrrrr} 
Date & Speed $(\mathbf{m} / \mathbf{s})$ & & Date & Speed $(\mathbf{m} / \mathbf{s})$ \\
\hline 1 & 0.56 & & 17 & 0.28 \\
2 & 0.28 & 18 & 0.83 \\
3 & 0.56 & 19 & 1.39 \\
4 & 0.56 & 20 & 1.11 \\
5 & 1.11 & 21 & 1.11 \\
6 & 0.83 & 22 & 0.83 \\
7 & 1.11 & 23 & 0.56 \\
8 & 1.94 & 24 & 0.83 \\
9 & 1.11 & 25 & 1.67 \\
10 & 0.83 & 26 & 1.94 \\
11 & 1.11 & 27 & 1.39 \\
12 & 1.39 & 28 & 0.83 \\
13 & 0.28 & 29 & 2.22 \\
14 & 0.56 & 30 & 1.67 \\
15 & 0.28 & 31 & 2.22 \\
16 & 0.28 & & \\
\hline
\end{tabular}

Table 5. Estimates of twoparameter Weibull by different methods.

\begin{tabular}{lrr} 
Method & Scale & Shape \\
\hline MLE & 1.1550 & 1.9081 \\
MOM & 1.1501 & 1.8456 \\
MRRS & 1.1636 & 1.8031 \\
Percentile 1 & 1.1100 & 1.8055 \\
Percentile 2 & 1.1816 & 2.1704 \\
\hline
\end{tabular}

\section{Conclusion}

Five different methods for the joint estimation of both scale and shape parameters of two-parameter Weibull distribution were reviewed in this article. A simulation study was conducted to compare the five methods based on bias, mean square error and variance of estimates. From simulation results, it was observed that MLE performs consistently better than MOM, MRRS, percentile method and 


\section{COMPARISON OF ESTIMATORS OF THE WEIBULL DISTRIBUTION}

improved percentile method and therefore MLE estimates are recommended to the practitioners.

\section{References}

Abernethy, R. B. (2006). (5 ${ }^{\text {th }}$ Ed.). The new Weibull handbook: reliability \& statistical analysis for predicting life, safety, risk, support costs, failures, and forecasting warranty claims, substantiation and accelerated testing, using Weibull, Log normal, crow-AMSAA, probit, and Kaplan-Meier models. North Palm Beach, FL: R. B. Abernethy.

Abernethy, R. B., Breneman, J. E., Medlin, C. H., \& Reinman, G. L. (1983). Weibull Analysis Handbook. Air Force Wright Aeronautical Laboratories Technical Report AFWAL-TR- 83-2079. Available at http://handle.dtic.mil/100.2/ADA143100.

Balakrishanan, N. \& Kateri, M. (2008). On the maximum likelihood estimation of parameters of Weibull distribution based on complete and censored data. Statistics \& Probability Letters, 78(17): 2971-2975.

Battacharya, P., \& Bhattacharjee, R. (2010). A study on Weibull distribution for estimating the parameters. Journal of Applied Quantitative Methods, 5(2): 234-241.

Celik, A. N. (2003). Energy output estimation for small-scale wind power generators using Weibull-representative wind data. Journal of Wind Engineering and Industrial Aerodynamics, 91: 693-707. doi: 10.1016/S0167-6105(02)00471-3

Dorvlo, A. S. (2002). Estimating wind speed distribution. Energy Conservation and Management, 43: 2311-2318.

Dubey, S. Y. D. (1967). Normal and Weibull distributions. Naval Research Logistics Quarterly, 14(1): 69-79. doi: 10.1002/nav.3800140107

Kantar, Y. M. \& Senoglu, B. (2008). A Comparative Study for the Location and Scale Parameters of the Weibull Distribution with a given Shape Parameter. Computers \& Geosciences, 34: 1900-1909.

Saralees, N., \& Firoozeh, H. (2011). An extension of the exponential distribution. Statistics - A Journal of Theoretical and Applied Statistics, 45(6): 543-558.

Saralees, N., \& Kotz, S. (2008). Strength modeling using Weibull distributions. Journal of Mechanical Science and Technology, 22: 1247-1254. 


\section{FLORENCE GEORGE}

Seki, T., \& Yokoyama, S. (1993). Simple and robust estimation of the Weibull parameters. Microelectronics Reliability, 33(1): 45-52.

Sharoon, H., Muhammad, Q. S., Muhammad, M., \& Kibria, B. M. G. (2012). A Note on Beta Inverse - Weibull Distribution. Communication in Statistics Theory and Methods, 42(2): 320-335.

Weisser, D. (2003). A wind energy analysis of Grenada: an estimation using the 'Weibull' density function. Renewable Energy; 28(11): 1803-1812. 